\title{
THE LEAST COMMON MULTIPLE OF CONSECUTIVE ARITHMETIC PROGRESSION TERMS
}

\author{
SHAOFANG HONG AND GUOYOU QIAN
}

\begin{abstract}
Let $k \geq 0, a \geq 1$ and $b \geq 0$ be integers. We define the arithmetic function $g_{k, a, b}$ for any positive integer $n$ by $g_{k, a, b}(n):=\frac{(b+n a)(b+(n+1) a) \cdots(b+(n+k) a)}{\operatorname{lcm}(b+n a, b+(n+1) a, \cdots, b+(n+k) a)}$. Letting $a=1$ and $b=0$, then $g_{k, a, b}$ becomes the arithmetic function introduced previously by Farhi. Farhi proved that $g_{k, 1,0}$ is periodic and that $k$ ! is a period. Hong and Yang improved Farhi's period $k !$ to $\operatorname{lcm}(1,2, \ldots, k)$ and conjectured that $\frac{\operatorname{lcm}(1,2, \ldots, k, k+1)}{k+1}$ divides the smallest period of $g_{k, 1,0}$. Recently, Farhi and Kane proved this conjecture and determined the smallest period of $g_{k, 1,0}$. For the general integers $a \geq 1$ and $b \geq 0$, it is natural to ask the interesting question: Is $g_{k, a, b}$ periodic? If so, then what is the smallest period of $g_{k, a, b}$ ? We first show that the arithmetic function $g_{k, a, b}$ is periodic. Subsequently, we provide detailed $p$-adic analysis of the periodic function $g_{k, a, b}$. Finally, we determine the smallest period of $g_{k, a, b}$. Our result extends the Farhi-Kane theorem from the set of positive integers to general arithmetic progressions.
\end{abstract}

\section{Introduction}

Many beautiful and important theorems about the arithmetic progression in number theory are known. Dirichlet's theorem [1] [11] and the Green-Tao theorem 9] are the two most famous examples. For some other results, see, for example, 4, 14, 15], 21] and 22. Meanwhile, the least common multiple of any given sequence of positive integers has received lots of attentions by many authors, see, for instance, [2, [3], [5- -17, 10- 13, [16, [19] and 20]. For the detailed background information about the least common multiple of finite arithmetic progressions, we refer the readers to [17.

Farhi [6], 7] investigated the least common multiple of a finite number of consecutive integers. Let $k \geq 0$ be an integer. It was proved in [6] and [7] that $\operatorname{lcm}(n, n+1, \ldots, n+k)$ is divisible by $n\left(\begin{array}{c}n+k \\ k\end{array}\right)$ and also divides $n\left(\begin{array}{c}n+k \\ k\end{array}\right) \operatorname{lcm}\left(\left(\begin{array}{l}k \\ 0\end{array}\right),\left(\begin{array}{l}k \\ 1\end{array}\right), \ldots,\left(\begin{array}{l}k \\ k\end{array}\right)\right)$. Farhi [6], 7] showed that the last equality holds if $k ! \mid(n+k+1)$. Farhi also introduced the arithmetic function $g_{k}$, which is defined for any positive integer $n$ by

$$
g_{k}(n):=\frac{n(n+1) \cdots(n+k)}{\operatorname{lcm}(n, n+1, \cdots, n+k)} .
$$

Farhi then proved that the sequence $\left\{g_{k}\right\}_{k=0}^{\infty}$ satisfies the recursive relation $g_{k}(n)=$ $\operatorname{gcd}\left(k !,(n+k) g_{k-1}(n)\right)$ for all positive integers $n$, where $\operatorname{gcd}(a, b)$ means the greatest common divisor of integers $a$ and $b$. Using this relation, we can easily show (by induction on $k$ ) that for any nonnegative integer $k$, the function $g_{k}$ is periodic of period $k$ !. This

Date: October 24, 2018.

2000 Mathematics Subject Classification. Primary 11B25, 11N13, 11A05.

Key words and phrases. arithmetic progression, least common multiple, $p$-adic valuation, arithmetic function, the smallest period,

The research was supported partially by National Science Foundation of China Grant \# 10971145 and by Program for New Century Excellent Talents in University Grant \# NCET-06-0785. 
is a result due to Farhi [7. Define $P_{k}$ to be the smallest period of the function $g_{k}$. Then Farhi's result says that $P_{k} \mid k$ !. Define $L_{0}:=1$ and for any integer $k \geq 1$, we define $L_{k}:=\operatorname{lcm}(1,2, \ldots, k)$. Hong and Yang [17] showed that $g_{k}(1) \mid g_{k}(n)$ for any nonnegative integer $k$ and any positive integer $n$. Consequently, using this result, they showed that $P_{k} \mid L_{k}$ for all positive integer $k$. This improves Farhi's period. In [17, Hong and Yang raised a conjecture stating that $\frac{L_{k+1}}{k+1}$ divides $P_{k}$ for all nonnegative integers $k$. From this conjecture, one can read that $k \mid P_{k}$ and $P_{k}=L_{k}$ if $k+1$ is a prime. Very recently, Farhi and Kane 8 found a proof of the Hong-Yang conjecture. Furthermore, Farhi and Kane determined the exact value of $P_{k}$, which solved the open problem posed by Farhi in [7].

Throughout this paper, let $\mathbb{Q}$ and $\mathbb{N}$ denote the field of rational numbers and the set of positive integers. Define $\mathbb{N}_{0}:=\mathbb{N} \cup\{0\}$. Let $k, b \in \mathbb{N}_{0}$ and $a \in \mathbb{N}$. We define the arithmetic function $g_{k, a, b}: \mathbb{N} \longrightarrow \mathbb{N}$ by

$$
g_{k, a, b}(n)=\frac{(b+n a)(b+(n+1) a) \cdots(b+(n+k) a)}{\operatorname{lcm}(b+n a, b+(n+1) a, \cdots, b+(n+k) a)} .
$$

Note that $g_{k, 1,0}=g_{k}$. It is natural to ask the following interesting question:

Problem 1.1. Let $k \geq 0, a \geq 1$ and $b \geq 0$ be integers. Is $g_{k, a, b}$ periodic and, if so, what is the smallest period of $g_{k, a, b}$ ?

Assume that $g_{k, a, b}$ is periodic and $P_{k, a, b}$ is the smallest period of $g_{k, a, b}$. Then we can use $P_{k, a, b}$ to give a formula for $\operatorname{lcm}(b+n a, b+(n+1) a, \cdots, b+(n+k) a)$ as follows: For any positive integer $n$, we have

$$
\operatorname{lcm}(b+n a, b+(n+1) a, \cdots, b+(n+k) a)=\frac{(b+n a)(b+(n+1) a) \cdots(b+(n+k) a)}{g_{k, a, b}\left(\langle n\rangle_{P_{k, a}, b}\right)},
$$

where $\langle n\rangle_{P_{k, a, b}}$ means the least nonnegative residue of $n$ modulo $P_{k, a, b}$. Therefore it is important to determine the exact value of $P_{k, a, b}$.

In this paper, we investigate the least common multiple of consecutive terms in arithmetic progressions. As usual, for any prime number $p$, we let $v_{p}$ be the normalized $p$-adic valuation of $\mathbb{Q}$, i.e., $v_{p}(a)=s$ if $p^{s} \| a$. For any real number $x$, by $\lfloor x\rfloor$ we denote the largest integer no more than $x$. Let $e_{p, k}:=\left\lfloor\log _{p} k\right\rfloor=\max _{1 \leq i \leq k}\left\{v_{p}(i)\right\}$ be the largest exponent of a power of $p$ that is at most $k$. We can now give the main result of this paper as follows.

Theorem 1.2. Let $k \geq 0, a \geq 1$ and $b \geq 0$ be integers. Then the arithmetic function $g_{k, a, b}$ is periodic, and if $\operatorname{gcd}(a, b)=1$, then its smallest period equals $Q_{k, a}$, where

$$
Q_{k, a}:=\frac{L_{k}}{\delta_{k, a} \cdot \prod_{\text {prime }} \prod_{q \mid \operatorname{gcd}\left(a, L_{k}\right)} q^{e_{q, k}}},
$$

and

$$
\delta_{k, a}:=\left\{\begin{array}{cl}
p^{e_{p, k},} & \text { if } p \nmid a \text { and } v_{p}(k+1) \geq e_{p, k} \text { for some prime } p \leq k, \\
1, & \text { otherwise. }
\end{array}\right.
$$

For $\operatorname{gcd}(a, b)>1$, its smallest period is equal to $Q_{k, a^{\prime}}$ with $a^{\prime}=\frac{a}{\operatorname{gcd}(a, b)}$.

Thus we answer completely Problem 1.1. Our result extends the Farhi-Kane theorem from the set of positive integers to general arithmetic progressions. 
The paper is organized as follows. In Section 2, by using a well-known result of Hua [18], we show that the arithmetic function $g_{k, a, b}$ is periodic; see Theorem 2.5. Then in Section 3, we provide detailed $p$-adic analysis to the periodic function $g_{k, a, b}$ and determine the smallest period of $g_{k, a, b}$. In the last section, we show Theorem 1.2 and give an example to illustrate the validity of Theorem 1.2.

\section{The periodicity of $g_{k, a, b}$}

Hong and Yang [17 proved that $L_{k}$ is a period of $g_{k}$. In this section, we introduce a new method to show that for any integers $k \geq 0, a \geq 1$ and $b \geq 0$, the arithmetic function $g_{k, a, b}$ is periodic, and particularly $L_{k}$ is also a period of $g_{k, a, b}$. First we need a well-known result of Hua. One can easily deduce this result by using the principle of inclusion-exclusion (see, for instance, Page 11 of [18]).

Lemma 2.1. 18 Let $a_{1}, a_{2}, \ldots, a_{n}$ be any $n$ positive integers. Then we have

$$
\operatorname{lcm}\left(a_{1}, a_{2}, \ldots, a_{n}\right)=a_{1} a_{2} \ldots a_{n} \cdot \prod_{r=2}^{n} \prod_{1 \leq i_{1}<\ldots<i_{r} \leq n}\left(\operatorname{gcd}\left(a_{i_{1}}, \ldots, a_{i_{r}}\right)\right)^{(-1)^{r-1}} .
$$

Lemma 2.2. Let $a_{1}, a_{2}, \ldots, a_{n}$ and $b_{1}, b_{2}, \ldots, b_{n}$ be any $2 n$ positive integers. Let $3 \leq t \leq$ $n$ be a given integer. If $\operatorname{gcd}\left(a_{i_{1}}, \ldots, a_{i_{t}}\right)=\operatorname{gcd}\left(b_{i_{1}}, \ldots, b_{i_{t}}\right)$ for any $1 \leq i_{1}<\ldots<i_{t} \leq n$, then we have

$$
\begin{aligned}
& \frac{a_{1} a_{2} \cdots a_{n}}{\operatorname{lcm}\left(a_{1}, a_{2}, \ldots, a_{n}\right)} \cdot \prod_{r=2}^{t-1} \prod_{1 \leq i_{1}<\ldots<i_{r} \leq n}\left(\operatorname{gcd}\left(a_{i_{1}}, \ldots, a_{i_{r}}\right)\right)^{(-1)^{r-1}} \\
= & \frac{b_{1} b_{2} \cdots b_{n}}{\operatorname{lcm}\left(b_{1}, b_{2}, \ldots, b_{n}\right)} \cdot \prod_{r=2}^{t-1} \prod_{1 \leq i_{1}<\ldots<i_{r} \leq n}\left(\operatorname{gcd}\left(b_{i_{1}}, \ldots, b_{i_{r}}\right)\right)^{(-1)^{r-1}} .
\end{aligned}
$$

Proof. If $\operatorname{gcd}\left(a_{i_{1}}, \ldots, a_{i_{t}}\right)=\operatorname{gcd}\left(b_{i_{1}}, \ldots, b_{i_{t}}\right)$ for any $1 \leq i_{1}<\ldots<i_{t} \leq n$, then we have $\operatorname{gcd}\left(a_{i_{1}}, \ldots, a_{i_{k}}\right)=\operatorname{gcd}\left(b_{i_{1}}, \ldots, b_{i_{k}}\right)$ for any $1 \leq i_{1}<\ldots<i_{k} \leq n$ and any $n \geq k \geq t$. Thus, by using Lemma 2.1, we get the result of Lemma 2.2 .

In particular, we have the following result.

Lemma 2.3. Let $a_{1}, a_{2}, \ldots, a_{n}$ and $b_{1}, b_{2}, \ldots, b_{n}$ be any $2 n$ positive integers. If for any $1 \leq i_{1}<i_{2}<i_{3} \leq n$, we have $\operatorname{gcd}\left(a_{i_{1}}, a_{i_{2}}, a_{i_{3}}\right)=\operatorname{gcd}\left(b_{i_{1}}, b_{i_{2}}, b_{i_{3}}\right)$, then

$$
\frac{1}{\prod_{1 \leq i<j \leq n} \operatorname{gcd}\left(a_{i}, a_{j}\right)} \cdot \frac{a_{1} a_{2} \cdots a_{n}}{\operatorname{lcm}\left(a_{1}, a_{2}, \ldots, a_{n}\right)}=\frac{1}{\prod_{1 \leq i<j \leq n} \operatorname{gcd}\left(b_{i}, b_{j}\right)} \cdot \frac{b_{1} b_{2} \cdots b_{n}}{\operatorname{lcm}\left(b_{1}, b_{2}, \ldots, b_{n}\right)} \text {. }
$$

Proof. Since $\operatorname{gcd}\left(a_{i_{1}}, a_{i_{2}}, a_{i_{3}}\right)=\operatorname{gcd}\left(b_{i_{1}}, b_{i_{2}}, b_{i_{3}}\right)$ for any $1 \leq i_{1}<i_{2}<i_{3} \leq n$, so we have $\operatorname{gcd}\left(a_{i_{1}}, \ldots, a_{i_{k}}\right)=\operatorname{gcd}\left(a_{i_{1}}, \ldots, a_{i_{k}}\right)$ for any $1 \leq i_{1}<\cdots<i_{k} \leq n$ and $k \geq 3$. By using Lemma 2.1, we get the conclusion of Lemma 2.3.

Notice that if $\operatorname{gcd}\left(a_{i}, a_{j}\right)=\operatorname{gcd}\left(b_{i}, b_{j}\right)$ for any $1 \leq i<j \leq n$, then $\operatorname{gcd}\left(a_{i_{1}}, a_{i_{2}}, a_{i_{3}}\right)=$ $\operatorname{gcd}\left(b_{i_{1}}, b_{i_{2}}, b_{i_{3}}\right)$ for any $1 \leq i_{1}<i_{2}<i_{3} \leq n$. It follows immediately from Lemma 2.3 that the following is true. 
Corollary 2.4. Let $a_{1}, a_{2}, \ldots, a_{n}$ and $b_{1}, b_{2}, \ldots, b_{n}$ be any $2 n$ positive integers. If $\operatorname{gcd}\left(a_{i}, a_{j}\right)=\operatorname{gcd}\left(b_{i}, b_{j}\right)$ for any $1 \leq i<j \leq n$, then we have

$$
\frac{a_{1} a_{2} \cdots a_{n}}{\operatorname{lcm}\left(a_{1}, a_{2}, \ldots, a_{n}\right)}=\frac{b_{1} b_{2} \cdots b_{n}}{\operatorname{lcm}\left(b_{1}, b_{2}, \ldots, b_{n}\right)} .
$$

We can now give the main result of this section. This also gives an alternative proof to the Hong-Yang period of the periodic function $g_{k}$ [17.

Theorem 2.5. Let $k \geq 0, a \geq 1$ and $b \geq 0$ be integers. Then the arithmetic function $g_{k, a, b}$ is periodic, and $L_{k}$ is a period of $g_{k, a, b}$.

Proof. Let $n$ be any positive integer. For any $0 \leq i<j \leq k$, we have

$$
\begin{aligned}
\operatorname{gcd}\left(b+\left(n+i+L_{k}\right) a, b+\left(n+j+L_{k}\right) a\right) & =\operatorname{gcd}\left(b+\left(n+i+L_{k}\right) a,(j-i) a\right) \\
& =\operatorname{gcd}(b+(n+i) a,(j-i) a) \\
& =\operatorname{gcd}(b+(n+i) a, b+(n+j) a) .
\end{aligned}
$$

Thus by Corollary 2.4, we obtain

$$
\begin{aligned}
& \frac{\left(b+\left(n+L_{k}\right) a\right)\left(b+\left(n+1+L_{k}\right) a\right) \cdots\left(b+\left(n+k+L_{k}\right) a\right)}{\operatorname{lcm}\left(b+\left(n+L_{k}\right) a, b+\left(n+1+L_{k}\right) a, \cdots, b+\left(n+k+L_{k}\right) a\right)} \\
& =\frac{(b+n a)(b+(n+1) a) \cdots(b+(n+k) a)}{\operatorname{lcm}(b+n a, b+(n+1) a, \cdots, b+(n+k) a)} .
\end{aligned}
$$

In other words, for any positive integer $n$, we have $g_{k, a, b}\left(n+L_{k}\right)=g_{k, a, b}(n)$ as desired.

Evidently, Theorem 2.5 gives an affirmative answer to the first part of Problem 1.1.

\section{3. $p$-Adic analysis of $g_{k, a, b}$}

Throughout this section, we always let $k \geq 0, a \geq 1$ and $b \geq 0$ be integers such that $\operatorname{gcd}(a, b)=1$. From the main result of previous section (Theorem 2.5), we know that the arithmetic function $g_{k, a, b}$ is periodic. Let $P_{k, a, b}$ denote the smallest period of $g_{k, a, b}$. Then by Theorem 2.5 we know that $P_{k, a, b}$ is a divisor of $L_{k}$. But the exact value of $P_{k, a, b}$ is still unknown. In this section, we will determine the exact value of $P_{k, a, b}$. We need some more notation. Let

$$
S_{k, a, b}(n):=\{b+n a, b+(n+1) a, \ldots, b+(n+k) a\}
$$

be any $k+1$ consecutive terms in the arithmetic progression $\{b+m a\}_{m \in \mathbb{N}_{0}}$. For a given prime number $p$, define $g_{p, k, a, b}(n):=v_{p}\left(g_{k, a, b}(n)\right)$. Since $g_{k, a, b}$ is a periodic function, $g_{p, k, a, b}$ is also a periodic function for each prime $p$ and $P_{k, a, b}$ is a period of $g_{p, k, a, b}$. Let $P_{p, k, a, b}$ be the smallest period of $g_{p, k, a, b}$. We have the following result.

Lemma 3.1. We have $P_{k, a, b}=\operatorname{lcm}_{p \text { prime }}\left(P_{p, k, a, b}\right)$.

Proof. Since for any $n \in \mathbb{N}$, we have that $v_{p}\left(g_{k, a, b}\left(n+P_{k, a, b}\right)\right)=v_{p}\left(g_{k, a, b}(n)\right)$, i.e., $P_{p, k, a, b} \mid P_{k, a, b}$ for each prime $p$. Hence we have $\operatorname{lcm}_{p}$ prime $\left(P_{p, k, a, b}\right) \mid P_{k, a, b}$.

Conversely, for any $n \in \mathbb{N}$, we have that $v_{p}\left(g_{k, a, b}\left(n+\operatorname{lcm}_{p}\right.\right.$ prime $\left.\left(P_{p, k, a, b}\right)\right)=v_{p}\left(g_{k, a, b}(n)\right)$ for each prime $p$. Thus, we have $g_{k, a, b}\left(n+\operatorname{lcm}_{p}\right.$ prime $\left.\left(P_{p, k, a, b}\right)\right)=g_{k, a, b}(n)$ for any $n \in \mathbb{N}$, that is, we have $P_{k, a, b} \mid \operatorname{lcm}_{p \text { prime }}\left(P_{p, k, a, b}\right)$. Therefore, we have $P_{k, a, b}=$ $\operatorname{lcm}_{p}$ prime $\left(P_{p, k, a, b}\right)$, as required. 
Hence we only need to compute $P_{p, k, a, b}$ for each prime $p$ to get the exact value of $P_{k, a, b}$. The following result is due to Farhi [6]. An alternative proof of it was given by Hong and Feng [12].

Lemma 3.2. 6] [12 Let $\left\{u_{i}\right\}_{i \in \mathbb{N}_{0}}$ be a strictly increasing arithmetic progression of nonzero integers and $k$ be any given non-negative integer. Then the integer $\operatorname{lcm}\left(u_{0}, u_{1}, \ldots\right.$, $\left.u_{k}\right)$ is a multiple of $\frac{u_{0} u_{1} \cdots u_{k}}{k !\left(\operatorname{gcd}\left(u_{0}, u_{1}\right)\right)^{k}}$.

Lemma 3.3. For any positive integer $n$, we have $g_{k, a, b}(n) \mid k !$.

Proof. Let $u_{i}=b+a(n+i)$ for $0 \leq i \leq k$. Then $\operatorname{gcd}\left(u_{0}, u_{1}\right)=1$ since $a$ and $b$ are coprime. So by Lemma 3.2 we know that there is an integer $A$ such that

$\operatorname{lcm}(b+n a, b+(n+1) a, \ldots, b+(n+k) a)=A \cdot \frac{(b+a n)(b+a(n+1)) \cdots(b+a(n+k)}{k !}$.

It then follows that $k !=A \cdot g_{k, a, b}(n)$.

It follows from Lemma 3.3 that $g_{p, k, a, b}(n)=v_{p}\left(g_{k, a, b}(n)\right)=0$ for each prime $p>k$ and any positive integer $n$. Hence we have $P_{p, k, a, b}=1$ for each prime $p>k$. So by Lemma 3.1, in order to determine the exact value of $P_{k, a, b}$, it suffices to compute the exact value of $P_{p, k, a, b}$ for all the primes $p$ such that $1<p \leq k$. First we consider the case that $p \mid a$ and $1<p \leq k$. Since $\operatorname{gcd}(a, b)=1$, we have $\operatorname{gcd}(p, b)=1$, and thus $\operatorname{gcd}(p, b+(n+i) a)=1$ for any integers $n \in \mathbb{N}$ and $0 \leq i \leq k$. Hence $\operatorname{gcd}\left(p, g_{k, a, b}(n)\right)=1$ for any integer $n \geq 1$, i.e., we have $g_{p, k, a, b}(n)=0$ for any integer $n \geq 1$ if $p \mid a$. Thus $P_{p, k, a, b}=1$ if $p \mid a$. We put these facts into the following lemma.

Lemma 3.4. Let $p$ be a prime such that either $p>k$ or $p \mid a$. Then we have $P_{p, k, a, b}=1$.

In what follows we treat the remaining case that $p \nmid a$ and $1<p \leq k$. Clearly we have

$$
\begin{aligned}
g_{p, k, a, b}(n) & =\sum_{m \in S_{k, a, b}(n)} v_{p}(m)-\max _{m \in S_{k, a, b}(n)} v_{p}(m) \\
& =\sum_{e \geq 1} \sum_{m \in S_{k, a, b}(n)}\left(1 \text { if } p^{e} \mid m\right)-\sum_{e \geq 1}\left(1 \text { if } p^{e} \text { divides some } m \in S_{k, a, b}(n)\right) \\
& =\sum_{e \geq 1} \#\left\{m \in S_{k, a, b}(n): p^{e} \mid m\right\}-\sum_{e \geq 1}\left(1 \text { if } p^{e} \text { divides some } m \in S_{k, a, b}(n)\right) \\
& =\sum_{e \geq 1} \max \left(0, \#\left\{m \in S_{k, a, b}(n): p^{e} \mid m\right\}-1\right) .
\end{aligned}
$$

Then we have the following lemmas:

Lemma 3.5. If $p \nmid a$ and $e>e_{p, k}$, then there is at most one element of $S_{k, a, b}(n)$ which is divisible by $p^{e}$.

Proof. Suppose that there exist two integers $i, j$ such that $p^{e} \mid b+(n+i) a$ and $p^{e} \mid$ $b+(n+j) a$, where $0 \leq i<j \leq k$, then we have $p^{e} \mid(j-i) a$. Since $\operatorname{gcd}(p, a)=1$, we get $p^{e} \mid(j-i)$. From it we deduce that $v_{p}(j-i) \geq e>e_{p, k}$. This is a contradiction. 
Lemma 3.6. Let $e$ be a positive integer. If $p \nmid a$, then any $p^{e}$ consecutive terms in the arithmetic progression $\{b+m a\}_{m \in \mathbb{N}_{0}}$ are pairwise incongruent modulo $p^{e}$. Furthermore, if $e \leq e_{p, k}$, then there is at least one element of $S_{k, a, b}(n)$ divisible by $p^{e}$.

Proof. Suppose that there exist two integers $i, j$ such that $b+(m+i) a \equiv b+(m+j) a$ $\left(\bmod p^{e}\right)$, where $m \geq 0$ and $0 \leq i<j \leq p^{e}-1$. Then $p^{e} \mid(j-i) a$. Since $\operatorname{gcd}(p, a)=1$, we have $p^{e} \mid(j-i)$. This is impossible. Thus the first part is true.

Now let $e \leq e_{p, k}$. Then $1 \leq p^{e} \leq k$. Hence $S_{k, a, b}(n)$ holds $p^{e}$ consecutive terms and one of which is divisible by $p^{e}$ by the above discussion. So the second part holds.

By Lemma 3.5, we know that all the terms in the right-hand side of (3.1) are 0 if $e>e_{p, k}$. By Lemma 3.6, there is at least one element divisible by $p^{e}$ in the set $S_{k, a, b}(n)$ if $e \leq e_{p, k}$. Therefore we obtain by (3.1)

$$
g_{p, k, a, b}(n)=\sum_{e=1}^{e_{p, k}} f_{e}(n),
$$

where $f_{e}(n):=\#\left\{m \in S_{k, a, b}(n): p^{e} \mid m\right\}-1$. Since $b+\left(n+i+p^{e}\right) a \equiv b+(n+i) a$ $\left(\bmod p^{e}\right)$ for any $i \in\{0,1, \ldots, k\}$, we have $f_{e}\left(n+p^{e}\right)=f_{e}(n)$. Therefore $p^{e}$ is a period of $f_{e}(n)$. Hence $f_{e}\left(n+p^{e_{p, k}}\right)=f_{e}(n)$ is true for each $e \in\left\{1, \ldots, e_{p, k}\right\}$. This implies that $g_{p, k, a, b}\left(n+p^{e_{p, k}}\right)=g_{p, k, a, b}(n)$. Consequently, $p^{e_{p, k}}$ is a period of $g_{p, k, a, b}(n)$. Thus $P_{p, k, a, b} \mid$ $p^{e_{p, k}}$. It follows immediately that the $P_{p, k, a, b}$ are relatively prime for different prime numbers $p$. But Lemma 3.1 and Lemma 3.4 tell us that $P_{k, a, b}=\operatorname{lcm}_{p}$ prime, $p \leq k, p \nmid a\left(P_{p, k, a, b}\right)$. Therefore we get the following result.

Lemma 3.7. We have

$$
P_{k, a, b}=\prod_{p \text { prime }, p \nmid a, p \leq k} P_{p, k, a, b},
$$

where $P_{p, k, a, b}$ satisfies that $P_{p, k, a, b} \mid p^{e_{p, k}}$.

According to Lemma 3.7, it suffices to compute the $p$-adic valuation of $P_{p, k, a, b}$ for the prime numbers $p$ satisfying $p \nmid a$ and $p \in(1, k]$. Now let us determine the $p$-adic valuation of $P_{k, a, b}$ for these prime numbers $p$.

Proposition 3.8. Let $a \geq 1$ and $b \geq 0$ be integers such that $\operatorname{gcd}(a, b)=1$. Let $k \geq 2$ be an integer and $p \in(1, k]$ be a prime number such that $p \nmid a$.

(i). If $v_{p}(k+1)<e_{p, k}$, then $v_{p}\left(P_{k, a, b}\right)=e_{p, k}$.

(ii). If $v_{p}(k+1) \geq e_{p, k}$, then $v_{p}\left(P_{k, a, b}\right)=0$.

Proof. (i). Since $p^{e_{p, k}}$ is a period of $g_{p, k, a, b}$, it suffices to prove that $p^{e_{p, k}-1}$ is not the period of $g_{p, k, a, b}$, from which it follows that $p^{e_{p, k}}$ is the smallest period of $g_{p, k, a, b}$. By (3.2), we have

$$
g_{p, k, a, b}(n)=\sum_{e=1}^{e_{p, k}} f_{e}(n)=\sum_{e=1}^{e_{p, k}-1} f_{e}(n)+f_{e_{p, k}}(n) .
$$

Since $p^{e_{p, k}-1}$ is a period of $\sum_{e=1}^{e_{p, k}-1} f_{e}(n)$, it is sufficient to prove that $p^{e_{p, k}-1}$ is not the period of $f_{e_{p, k}}(n)$. We claim that there exists a positive integer $n_{0}$ such that $f_{e_{p, k}}\left(n_{0}+\right.$ $\left.p^{e_{p, k}-1}\right)=f_{e_{p, k}}\left(n_{0}\right)-1$. 
By $v_{p}(k+1)<e_{p, k}$, we deduce that $p^{e_{p, k}} \nmid(k+1)$ and $p^{e_{p, k}} \leq k$. We can suppose that $k+1 \equiv l\left(\bmod p^{e_{p, k}}\right)$ for some $1 \leq l \leq p^{e_{p, k}}-1$. We divide the proof of part (i) into the following two cases:

CASE $1.1 \leq l \leq p^{e_{p, k}}-p^{e_{p, k}-1}$. Since $p \nmid a$, we can always find a suitable $n_{0}$ such that $b+n_{0} a \equiv 0\left(\bmod p^{e_{p, k}}\right)$. Consider the following two sets:

$S_{k, a, b}\left(n_{0}\right)=\left\{b+n_{0} a, \ldots, b+\left(n_{0}+p^{e_{p, k}-1}-1\right) a, b+\left(n_{0}+p^{e_{p, k}-1}\right) a, \ldots, b+\left(n_{0}+k\right) a\right\}$

and

$$
\begin{aligned}
& S_{k, a, b}\left(n_{0}+p^{e_{p, k}-1}\right)=\left\{b+\left(n_{0}+p^{e_{p, k}-1}\right) a, \ldots, b+\left(n_{0}+k\right) a,\right. \\
& \left.b+\left(n_{0}+k+1\right) a, \ldots, b+\left(n_{0}+k+p^{e_{p, k}-1}\right) a\right\} .
\end{aligned}
$$

Now $\left\{b+\left(n_{0}+p^{e_{p, k}-1}\right) a, \ldots, b+\left(n_{0}+k\right) a\right\}$ is the intersection of $S_{k, a, b}\left(n_{0}\right)$ and $S_{k, a, b}\left(n_{0}+\right.$ $\left.p^{e_{p, k}-1}\right)$. So to compare the number of terms divisible by $p^{e_{p, k}}$ in the set $S_{k, a, b}\left(n_{0}\right)$ with the number of terms divisible by $p^{e_{p, k}}$ in the set $S_{k, a, b}\left(n_{0}+p^{e_{p, k}-1}\right)$, it suffices to compare the number of terms divisible by $p^{e_{p, k}}$ in the set $\left\{b+n_{0} a, \ldots, b+\left(n_{0}+p^{e_{p, k}-1}-1\right) a\right\}$ with the number of terms divisible by $p^{e_{p, k}}$ in the set $\left\{b+\left(n_{0}+k+1\right) a, \ldots, b+\left(n_{0}+\right.\right.$ $\left.\left.k+p^{e_{p, k}-1}\right) a\right\}$. By Lemma 3.6, any $p^{e_{p, k}}$ consecutive terms in the arithmetic progression $\{b+m a\}_{m \in \mathbb{N}_{0}}$ are pairwise incongruent modulo $p^{e_{p, k}}$. Thus the terms divisible by $p^{e_{p, k}}$ in the arithmetic progression $\{b+m a\}_{m \in \mathbb{N}_{0}}$ must be of the form $b+\left(n_{0}+t p^{e_{p, k}}\right) a, t \in \mathbb{Z}$. Since $k+1 \equiv l\left(\bmod p^{e_{p, k}}\right)$ and $1 \leq l \leq p^{e_{p, k}}-p^{e_{p, k}-1}$, we have $k+j \equiv l+j-1 \not \equiv 0$ $\left(\bmod p^{e_{p, k}}\right)$ for all $\left.1 \leq j \leq p^{e_{p, k}-1}\right)$. Hence $p^{e_{p, k}} \nmid\left(b+\left(n_{0}+k+j\right) a\right.$ for all $1 \leq j \leq p^{e_{p, k}-1}$. Thus all the elements in the set $\left\{b+\left(n_{0}+k+1\right) a, \ldots, b+\left(n_{0}+k+p^{e_{p, k}-1}\right) a\right\}$ are not divisible by $p^{e_{p, k}}$. On the other hand, since $b+a n_{0} \equiv 0\left(\bmod p^{e_{p, k}}\right)$, it follows from Lemma 3.6 that there is exactly one term in the set $\left\{b+n_{0} a, b+\left(n_{0}+1\right) a, \ldots, b+\left(n_{0}+\right.\right.$ $\left.\left.p^{e_{p, k}-1}-1\right) a\right\}$ which is divisible by $p^{e_{p, k}}$. Therefore the number of terms divisible by $p^{e_{p, k}}$ in the set $S_{k, a, b}\left(n_{0}+p^{e_{p, k}-1}\right)$ is equal to the number of terms divisible by $p^{e_{p, k}}$ in the set $S_{k, a, b}\left(n_{0}\right)$ minus one. Namely, $f_{e_{p, k}}\left(n_{0}+p^{e_{p, k}-1}\right)=f_{e_{p, k}}\left(n_{0}\right)-1$ as required. The claim is proved in this case.

CASE 2. $p^{e_{p, k}}-p^{e_{p, k}-1}<l \leq p^{e_{p, k}}-1$. Since $p \nmid a$, it is easy to see that there is a positive integer $n_{0}$ such that $b+\left(n_{0}+p^{e_{p, k}-1}-1\right) a \equiv 0\left(\bmod p^{e_{p, k}}\right)$. As in the discussion of Case 1, to compare the number of terms divisible by $p^{e_{p, k}}$ in the set $S_{k, a, b}\left(n_{0}\right)$ with the number of terms divisible by $p^{e_{p, k}}$ in the set $S_{k, a, b}\left(n_{0}+p^{e_{p, k}-1}\right)$, it suffices to compare the number of terms divisible by $p^{e_{p, k}}$ in the set $\left\{b+n_{0} a, \ldots, b+\left(n_{0}+p^{e_{p, k}-1}-1\right) a\right\}$ with the number of terms divisible by $p^{e_{p, k}}$ in the set $\left\{b+\left(n_{0}+k+1\right) a, \ldots, b+\left(n_{0}+k+p^{e_{p, k}-1}\right) a\right\}$. From $b+\left(n_{0}+p^{e_{p, k}-1}-1\right) a \equiv 0\left(\bmod p^{e_{p, k}}\right)$ one can deduce that the terms divisible by $p^{e_{p, k}}$ in the arithmetic progression $\{b+m a\}_{m \in \mathbb{N}_{0}}$ must be of the form $b+\left(n_{0}+p^{e_{p, k}-1}-1+\right.$ $\left.t p^{e_{p, k}}\right) a$ with $t \in \mathbb{Z}$. Since $k+1 \equiv l\left(\bmod p^{e_{p, k}}\right)$ for some $p^{e_{p, k}}-p^{e_{p, k}-1}<l \leq p^{e_{p, k}}-1$, we have $p^{e_{p, k}}-p^{e_{p, k}-1}+1 \leq l+j-1 \leq p^{e_{p, k}}+p^{e_{p, k}-1}-2$ and so $k+j \equiv l+j-1 \not \equiv p^{e_{p, k}-1}-1$ $\left(\bmod p^{e_{p, k}}\right)$ for all $1 \leq j \leq p^{e_{p, k}-1}$. It follows that for all $1 \leq j \leq p^{e_{p, k}-1}$, we have $p^{e_{p, k}} \nmid\left(b+\left(n_{0}+k+j\right) a\right)$. That is, there does not exist an integer divisible by $p^{e_{p, k}}$ in the set $\left\{b+\left(n_{0}+k+1\right) a, \ldots, b+\left(n_{0}+k+p^{e_{p, k}-1}\right) a\right\}$. But the term $b+\left(n_{0}+p^{e_{p, k}-1}-1\right) a$ is the only term divisible by $p^{e_{p, k}}$ in the set $\left\{b+n_{0} a, b+\left(n_{0}+1\right) a, \ldots, b+\left(n_{0}+p^{e_{p, k}-1}-1\right) a\right\}$. Thus the number of terms divisible by $p^{e_{p, k}}$ in the set $S_{k, a, b}\left(n_{0}+p^{e_{p, k}-1}\right)$ equals the number of terms divisible by $p^{e_{p, k}}$ in the set $S_{k, a, b}\left(n_{0}\right)$ minus one. Hence the desired result $f_{e_{p, k}}\left(n_{0}+p^{e_{p, k}-1}\right)=f_{e_{p, k}}\left(n_{0}\right)-1$ follows immediately. The proof of the claim is complete.

From the claim we deduce immediately that $p^{e_{p, k}-1}$ is not a period of $g_{p, k, a, b}$. Thus $p^{e_{p, k}}$ is the smallest period of $g_{p, k, a, b}$. It follows that $v_{p}\left(P_{k, a, b}\right)=e_{p, k}$ as desired. 
(ii). By Lemma 3.7, we know that to prove part (ii), it is sufficient to prove that $v_{p}\left(P_{q, k, a, b}\right)=0$ for each prime $q$ with $q \leq k$ and $q \nmid a$. For any prime $q$ different from $p$, since $P_{q, k, a, b} \mid q^{e_{q, k}}$, we then have $v_{p}\left(P_{q, k, a, b}\right)=0$. In what follows we deal with the remaining case $q=p$.

From $v_{p}(k+1) \geq e_{p, k}$, we deduce that $p^{e_{p, k}} \mid(k+1)$ and $p^{e} \mid(k+1)$ for each $e \in\left\{1, \ldots, e_{p, k}\right\}$. By Lemma 3.6, any $p^{e}$ consecutive terms in the arithmetic progression $\{b+m a\}_{m \in \mathbb{N}_{0}}$ are pairwise incongruent modulo $p^{e}$ since $p \nmid a$. Hence for each $e \in$ $\left\{1, \ldots, e_{p, k}\right\}$, there are exactly $\frac{k+1}{p^{e}}$ terms divisible by $p^{e}$ in any $k+1$ consecutive terms of the arithmetic progression $\{b+m a\}_{m \in \mathbb{N}_{0}}$. So we have that $f_{e}(n)=\frac{k+1}{p^{e}}-1$ for each $e \in\left\{1, \ldots, e_{p, k}\right\}$. In other words, for every $n \in \mathbb{N}$, we have $f_{e}(n+1)=f_{e}(n)$. It then follows from (3.2) that for every $n \in \mathbb{N}$, we have $g_{p, k, a, b}(n+1)=g_{p, k, a, b}(n)$. Thus $P_{p, k, a, b}=1$ and $v_{p}\left(P_{k, a, b}\right)=0$. Therefore part (ii) is proved.

\section{Proof of Theorem $\mathbf{1 . 2}$}

In this section, we first prove Theorem 1.2.

Proof of Theorem 1.2: By Theorem 2.5, we know that $g_{k, a, b}$ is periodic. Denote by $P_{k, a, b}$ its smallest period. Let first $\operatorname{gcd}(a, b)=1$. Then by Lemma 3.7 , for any prime $p$ such that $p \mid a$, we have $v_{p}\left(P_{k, a, b}\right)=0$. For any prime $p$ satisfying $p \nmid a$ and $p \leq k$, we have by Lemma 3.7, $P_{p, k, a, b}=p^{v_{p}\left(P_{p, k, a, b}\right)}=p^{v_{p}\left(P_{k, a, b}\right)}$. So by Proposition 3.8 we infer that

$$
P_{k, a, b}=\prod_{p \text { prime }, p \leq k} p^{e_{p}(k, a)},
$$

where

$$
e_{p}(k, a):=\left\{\begin{aligned}
0, & \text { if } v_{p}(k+1) \geq e_{p, k} \text { or } p \mid a \\
e_{p, k}, & \text { otherwise. }
\end{aligned}\right.
$$

Using the integer $L_{k}$, we obtain immediately that $P_{k, a, b}=Q_{k, a}$ as required, where $Q_{k, a}$ is defined as in (1.1).

Now let $\operatorname{gcd}(a, b)>1$. If $\operatorname{gcd}(a, b)=d$ and $a=d a^{\prime}$ and $b=d b^{\prime}$, then $\operatorname{gcd}\left(a^{\prime}, b^{\prime}\right)=1$ and we can easily check that $g_{k, a, b}(n)=d^{k} g_{k, a^{\prime}, b^{\prime}}(n)$ for any $n \in \mathbb{N}$. From this one can easily derive that the periodic functions $g_{k, a, b}$ and $g_{k, a^{\prime}, b^{\prime}}$ have the same smallest period, i.e., $P_{k, a, b}=P_{k, a^{\prime}, b^{\prime}}$. But the result for the case $\operatorname{gcd}(a, b)=1$ applied to the function $g_{k, a^{\prime}, b^{\prime}}$ gives us that $P_{k, a^{\prime}, b^{\prime}}=Q_{k, a^{\prime}}$, with $Q_{k, a^{\prime}}$ defined as in (1.1). Thus the desired result $P_{k, a, b}=Q_{k, a^{\prime}}$ follows immediately. This completes the proof of Theorem 1.2.

It was proved by Farhi and Kane [8] that there is at most one prime $p \leq k$ such that $v_{p}(k+1) \geq e_{p, k}$. We noticed that such a prime $p$ was given in Proposition 3.3 of [8] without the condition $p \leq k$, but such restriction condition is clearly necessary because otherwise Proposition 3.3 of [8] would not be true. For example, letting $p$ be any prime number greater than $k+1$ gives us $v_{p}(k+1)=0=e_{p, k}$. Comparing the smallest period $P_{k, a, b}$ of the function $g_{k, a, b}$ with the smallest period $P_{k}$ of the function $g_{k}=g_{k, 1,0}$, we arrive at the relation between $P_{k, a, b}$ and $P_{k}$ as follows:

$$
P_{k, a, b}=\frac{P_{k}}{\prod_{\text {prime }} \mid \operatorname{gcd}\left(a^{\prime}, P_{k}\right)} p^{e_{p, k}}
$$

where $a^{\prime}=\frac{a}{\operatorname{gcd}(a, b)}$. From this one can read that $P_{k, a, b}=P_{k}$ if $a \mid b$. 
Finally, we give an application of Theorem 1.2 as the conclusion of this paper.

Example 4.1. Let us consider the least common multiple of any $k+1$ consecutive positive odd numbers. To study this problem, we define arithmetic function $h_{k}$ by

$$
h_{k}(n):=\frac{(2 n+1) \cdot(2 n+3) \cdots(2 n+2 k+1)}{\operatorname{lcm}(2 n+1,2 n+3, \cdots, 2 n+2 k+1)} \quad(n \in \mathbb{N})
$$

By Theorem 1.2, we know that $h_{k}$ is periodic and for any integer $k \geq 2$, the exact period $R_{k}$ of $h_{k}$ is given by $R_{k}=\frac{L_{k}}{2^{e, k} \cdot D_{k}}$, where

$$
D_{k}=\left\{\begin{aligned}
p^{e_{p, k},} & \text { if } v_{p}(k+1) \geq e_{p, k} \text { for some odd prime } p \leq k \\
1, & \text { otherwise. }
\end{aligned}\right.
$$

\section{Acknowledgment}

The authors would like to thank Professor Smyth and the referees for their careful reading of the manuscript and helpful suggestions which improved its presentation.

\section{REFERENCES}

[1] T.M. Apostol, Introduction to analytic number theory, Springer-Verlag, New York, 1976.

[2] G. Bachman and T. Kessler, On divisibility properties of certain multinomial coefficients II, J. Number Theory 106 (2004), 1-12.

[3] P. Bateman, J. Kalb and A. Stenger, A limit involving least common multiples, Amer. Math. Monthly 109 (2002), 393-394.

[4] M.A. Bennett, N. Bruin, K. Györy and L. Hajdu, Powers from products of consecutive terms in arithmetic progression, Proc. London Math. Soc. 92 (2006), 273-306.

[5] J. Cilleruelo, The least common multiple of a quadratic sequence, arXiv:1001.3438

[6] B. Farhi, Minoration non triviales du plus petit commun multiple de certaines suites finies d'entiers, C.R. Acad. Sci. Paris, Ser. I 341 (2005), 469-474.

[7] B. Farhi, Nontrivial lower bounds for the least common multiple of some finite sequences of integers, J. Number Theory 125 (2007), 393-411.

[8] B. Farhi and D. Kane, New results on the least common multiple of consecutive integers, Proc. Amer. Math. Soc. 137 (2009), 1933-1939.

[9] B. Green and T. Tao, The primes contain arbitrarily long arithmetic progressions, Ann. of Math. (2) 167 (2008), 481-547.

[10] D. Hanson, On the product of the primes, Canad. Math. Bull. 15 (1972), 33-37.

[11] G.H. Hardy and E.M. Wright, An introduction to the theory of numbers, Fourth Edition, Oxford University Press, London, 1960.

[12] S. Hong and W. Feng, Lower bounds for the least common multiple of finite arithmetic progressions, C.R. Acad. Sci. Paris, Ser. I 343 (2006), 695-698.

[13] S. Hong and S.D. Kominers, Further improvements of lower bounds for the least common multiple of arithmetic progressions, Proc. Amer. Math. Soc. 138 (2010), 809-813.

[14] S. Hong and K.S. Enoch Lee, Asymptotic behavior of eigenvalues of reciprocal power LCM matrices, Glasgow Math. J. 50 (2008), 163-174.

[15] S. Hong and R. Loewy, Asymptotic behavior of eigenvalues of greatest common divisor matrices, Glasgow Math. J. 46 (2004), 551-569.

[16] S. Hong and Y. Yang, Improvements of lower bounds for the least common multiple of finite arithmetic progressions, Proc. Amer. Math. Soc. 136 (2008), 4111-4114.

[17] S. Hong and Y. Yang, On the periodicity of an arithmetical function, C.R. Acad. Sci. Paris, Ser. I 346 (2008), 717-721.

[18] L.-K. Hua, Introduction to number theory, Springer-Verlag, Berlin Heidelberg, 1982.

[19] G. Myerson and J. Sander, What the least common multiple divides II, J. Number Theory 61 (1996), 67-84.

[20] M. Nair, On Chebyshev-type inequalities for primes, Amer. Math. Monthly 89 (1982), 126-129.

[21] N. Saradha, Squares in products with terms in an arithmetic progression, Acta Arith. 86 (1998), $27-43$. 
[22] N. Saradha and T.N. Shorey, Almost squares in arithmetic progression, Compositio Math. 138 (2003), 73-111.

Mathematical College, Sichuan University, Chengdu 610064, P.R. China

E-mail address: sfhong@scu.edu.cn, sfhong09@gmail.com, s-f.hong@tom.com, hongsf02@yahoo.com

Mathematical College, Sichuan University, Chengdu 610064, P.R. China

E-mail address: qiangy1230@gmail.com, qiangy1230@163.com, qiangy0901@tom.com 